\title{
A METHOD OF MEASURING THERMAL STABILITY IN DELTA-DOPING
}

\author{
Alfred Zehe ${ }^{\mathbf{a}, \mathbf{b}}$, Eusebio Torres-Tapia ${ }^{\mathbf{a}}$, Araceli Ramírez ${ }^{\mathbf{b}}$ \\ ${ }^{a}$ Facultad de Ciencias Fisicas, Universidad Nacional Mayor de San Marcos Ap. Postal 14-0149, Lima 14, Perú. \\ ${ }^{b}$ Facultad de Ciencias Electrónicas, Benemérita Universidad Autónoma de Puebla, Ap. Postal 1505, 72000 Puebla, México.
}

\begin{abstract}
A new superlattice in semiconductors is discussed which allows to determine the geometrical stability and precision of deita-doping layers in respect to migration and diffusion effects, and even a technological evaluation of the production device. This structure acts as inner probe in doping superlattices quite as the known case of enlarged wells in composition superlattices does. Donor - acceptor - pair luminescence radiation is used, which in its dependence on the pair separation distance reflects the geometrical arrangement of neighboring deltadoped layers provided both donors and acceptors are present in each sheet.
\end{abstract}

PACS: 29.40.-n; 29.40.Wk

Keywords: Superlattice, planar doping, luminescence, donor-acceptor pair, thermal stability, geometrical arrangement.

\section{Resumen}

Se discute una superred novedosa, que permite la determinación de la estabilidad geométrica y precisión de capas delta-dopadas con respecto a efectos de difusión y migración, así como la evaluación tecnológica de la producción de dispositivos. Esta estructura nanométrica actúa como sonda interna en copamiento de superredes, semejante al caso conocido de los "pozos ensanchados" en superredes de composición. Se usa la luminiscencia entre pares donador-aceptor, la cual depende de la distancia de separación que refleja el arreglo geométrico de capas delta-dopadas vecinas, siempre que existan donadores y aceptores en las capas correspondientes.

Palabras claves: Superred, dopamiento bidimensional, luminiscencia, pares donador-aceptor, estabilidad térmica, arreglo geométrico.

\section{Introducción}

Many properties of nanometer structures in semiconductors depend on the atomic scale smoothness of interfaces. Specialized technologies, like $\mathrm{MBE}$ and MO-CVD have brought about a high level of control of the film growth process with atomic scale precision, requiring interface analytical tools with the same accuracy. Single monolayer wellsize fluctuations of heterostructure quantum wells and composition superlattices, like GaAs/GaAlAsstructures, have been studied by use of excitonic luminescence radiation as early as in 1981, relating line width and also line splitting to intralayer fluctuations of one monolayer [1-3].

Nevertheless the existence of radiative luminescence transitions which carry crystal site information is well documented as Donor-AcceptorPair (DAP) radiation in bulk semiconductors [4,5]. Due to a dependence on $R$, the separation distance between pair forming donor and acceptor ions in real space, DAP transitions between voluntarily arranged doping sheets must reflect their mutual position.

The physical properties of doping superlattices have extensively been studied and reviewed [6,7]. Recent progress in MBE technology enabled the growth and characterization of Delta-doped crystals with sheet doping concentrations of about $10^{12-13} \mathrm{~cm}^{-2}$ $[8,10]$, which are favored by a series of interesting applications [11-13]. Nevertheless fluctuations of doping elements out of the contemplated sheet cause sub-optimal device operation.

Such fluctuations might be the consequence of one out of several reasons: diffusion during growth or due to the later operation of the structure, migration, radiation induced knocking-out of originally well placed atoms, or just the insufficient dominance of the doping and growth technology.

In the present paper the formation and excitation of DAPs within delta-doped layers, and between them, is used as an inner probe in order to detect sheet doping fluctuations down to one atomic layer 
dimensions, as well as the study of radiation hardness of such structures. The proposed structure is called Pair Doped $\Delta \mathrm{i} \Delta \mathrm{i}$ - Superlattice (PD- $\Delta \mathrm{i} \Delta \mathrm{i}$-SL), where the $\Delta$-symbol indicates the presence of both donors and acceptors within the same sheet. The superlattice contains two or more doping layers, separated by intrinsic bulk material.

\section{Delta Doping}

Delta-doping (also sheet-, spike-, or atomic plane doping) during the MBE growth of GaAs has been proposed by several authors $[14,15]$. Using Silicon as doping element, and a special growth regime (interrupted growth) PLOOG [14] reported sheet doping concentrations exceeding $10^{13} \mathrm{~cm}^{-2}$. A symmetric V-shaped potential well (Fig. 1) is created by the atomic-plane doping contrary to the asymmetric potential known from the classical SiMOSFET used for the study of two-dimensional physics during the 1980's. By virtue of the sheet doping concentration the potential depth can be controlled in a wide range. The energy levels in such a $\mathrm{V}$-shaped potential well have been calculated recently [16]. It was shown that the ground level $E_{0}$ moved up when the potential well depth $V_{0}$ was increased by a higher sheet-doping concentration.

Of course the potential edges at $\pm \mathrm{a}$ in Fig. 2 are smeared out for a better approximation of the more realistic potential, but as can be taken from Fig. 3, the ground energy state $E_{0}$ is deep inside the quantum well preventing remarkable effects from the abrupt edges.

It is clear that fluctuations of doping elements around the proposed atomic plane for sheet doping would influence the features of the potential, decreasing $V_{0}$ and increasing $2 a$. This effects 'per se' could be useful in order to detect sheet doping fluctuations, although a more precise treatment of the $V$-shaped quantum well would be necessary. No measurements are known yet.

\section{The Pair Doped $\Delta \mathrm{i} \Delta \mathrm{I}$ Superlattice as Inner Probe}

Provided the atomic-plane doping contains both donor and acceptor elements of concentration $D$ and A respectively, donor-acceptor pairs will form following a statistical distribution quite as in bulk semiconductors, but with the restriction of ion connection lines to the plane of doping. That means, instead of the bulk donor-acceptor pair distribution function, $G(R),[17]$, a two dimensional pair distribution function $G^{(2 d)}(R)$ has to be applied:
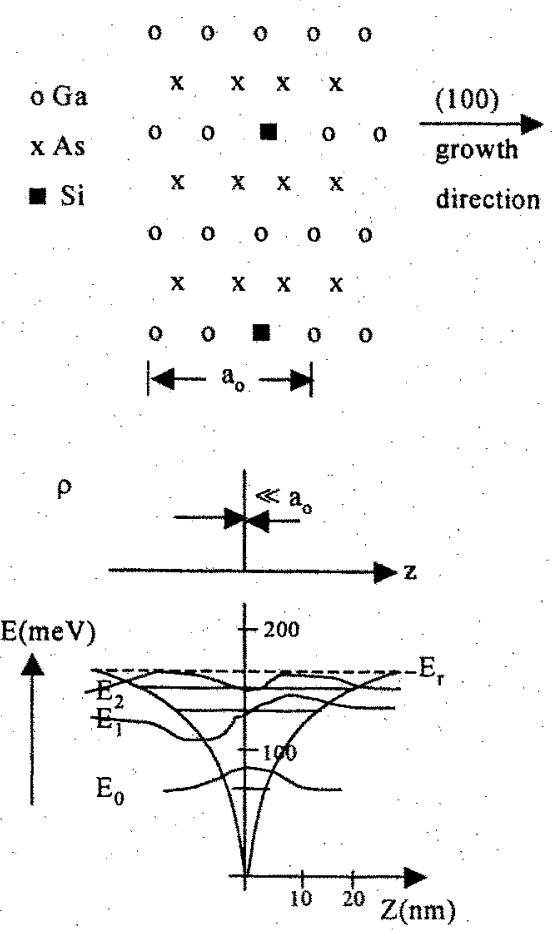

Fig. 1. Atomic-plane doping of GaAs with Si donors (top). A V-shaped potential well is formed (bottom). (after PLOOG [14]).

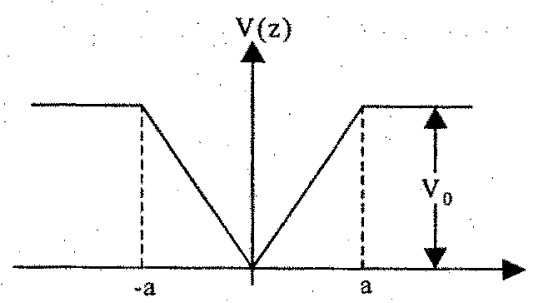

Fig, 2. Symmetric $V$-shaped potential well, $V(z)$; with depth $\mathrm{V}_{0}$ and width $2 \mathrm{a}$.

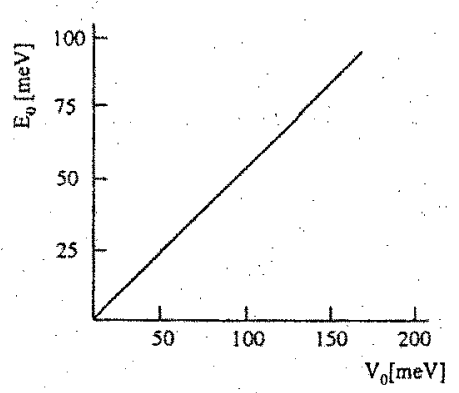

Fig. 3. Ground state energy, $E_{0}$, as function of potential well depth Vo, and $2 a=50 \AA$ (after ZEHE et al. [16]).

$G(R)=4 \pi R^{2} D \exp \left(-4 \pi R^{3} D / 3\right)$ 
$G^{(2 d)}(R)=2 \pi R D^{(2 d)} \exp \left(-\pi R^{2} D^{(2 d)}\right)$

Those formulas are based on the assumption here, that the probing acceptor concentration $\mathrm{A}$ is much lower than the chosen (operating) donor concentration $\mathrm{D}$.

Let $C(R) d^{3} R$ be the probability for a donor to be situated at a distance $R$ in the element $d^{3} R$ from a reference acceptor. The probability $G(R) d^{3} R$ to be this the next neighbor of the reference acceptor, is determined by the probability

$$
1-\int_{R^{\prime}<R} G\left(R^{\prime}\right) d^{3} R
$$

that no donor will be found at a distance $R^{\prime}<R$ giving for the distribution function $G(R)$ the integral equation

$$
G(R)=C(R)\left\{1-\int_{R^{\prime}<R} G\left(R^{\prime}\right) d^{3} R\right\}
$$

The solution of eq. (4), when $C(R)=D$ (homogeneously doped sample), is easily shown to be eq. (1). In the case of only one doped sheet, donor-acceptor pairs form within the same layer. With a sheet doping concentration of $\mathrm{D}^{(2 \mathrm{~d})}$, from eq. (4) follows the result for the pair distribution function in a sheet as given in eq. (2).

The Fig. 4 compares the DAP-distribution functions for bulk and sheet doping. Only the Rdependence is of interest, not the absolute peak value. It is clear that at comparable doping concentrations the chances of a certain acceptor to find a pairing donor are restricted in the sheet as compared to the bulk. A higher number of distant pairs is an expected effect then.

$$
C(R)=D^{(2 d)} \Theta(R-s)
$$

Annealing a sheet-doping structure long enough would cause the two-dimensional DAP-distribution function to transform into a bulk-like DAPdistribution function. But many diffusion steps would be necessary of both donors and acceptors to perform this transformation.

Introducing now at a separation distance $s$ (which must correlate with the effective Bohr radius $a^{*}$ of the donor and acceptor) a second Delta-doping sheet of the same kind as the first one is, a new situation for the donor-acceptor pairing is created: While close pairs within each of the doped sheets would not detect any difference, distant pairs will. For some acceptor atom in sheet number 1 there might be a donor in the probing sheet number 2 closer to it as if probed only within the same sheet.

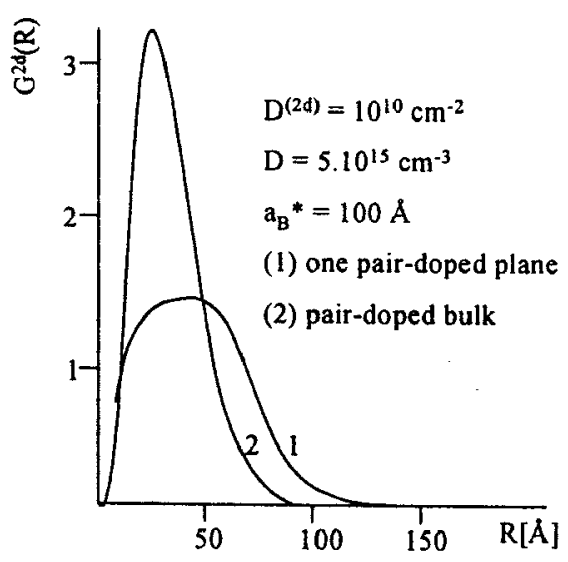

Fig. 4. Bulk doping concentration of donors $D=5 \cdot 10^{15} \mathrm{~cm}^{-3}$. Sheet doping concentration of donors used in the calculus is $\mathrm{D}^{(2 \mathrm{~d})}=10^{10} \mathrm{~cm}^{-2}$.

Using Fig. 5 as reference in order to calculate the pair-distribution function for the new situation, the result is given in eq. (7). As soon as the pair separation distance, $R$, hits the distance, $s$, of the probing sheet, an additional number of DAP's is created to the debit of more distant pairs in the original (operation) sheet. This effect is shown in Fig. 6 for the case of a probing superlattice (where of course any of the sheets could be considered as the operating delta-doped atomic plane).

In this case of a layered structure, we have at the distance $s$ from a reference acceptor a layer with the density per area $\mathrm{D}^{(2 \mathrm{~d})}$ of donors, and we write (see Fig. 5)

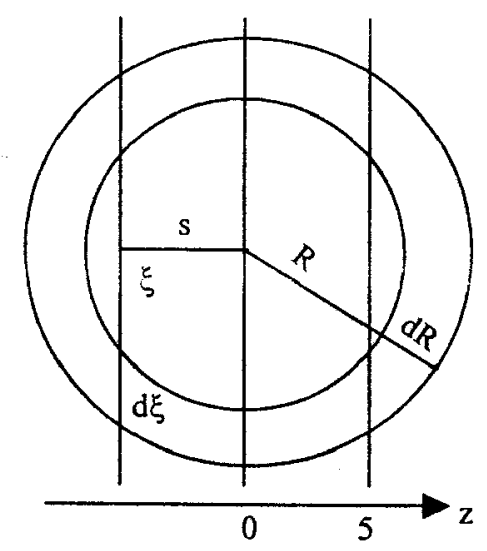

Fig. 5. Pair doped $\Delta \mathrm{i} \Delta \mathrm{i}-$ superlattice with several probing sheets in respect to the central sheet at $\mathrm{z}=0$. 


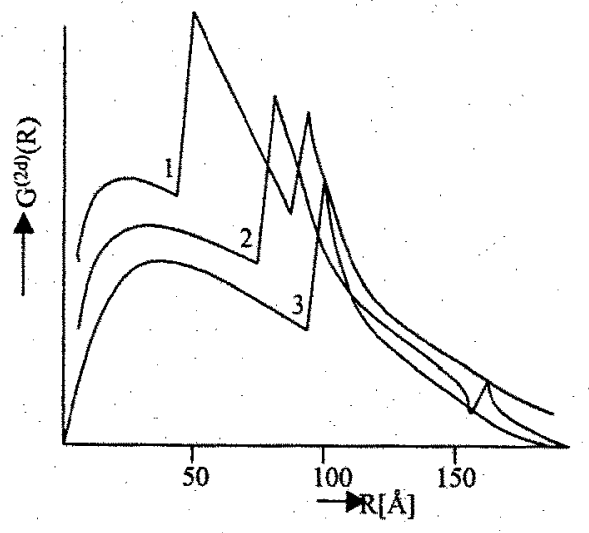

Fig. 6. Pair distribution function for a double layer system, where at a distance $s$ from the operating sheet appears a probing sheet of the same kind. Curves are vertically displaced for more clarity. (1) $\mathrm{s}=50 \AA$, (2) $\mathrm{s}=75 \AA$, (3) $\mathrm{s}=$ $100 \AA$.

The probability $C(R) d R$ to find a donor at the distance $R$ in the interval $d R$ is given by the solid angle average of $\mathrm{C}(\mathrm{R})$. The contribution from a probing layer at a distances is

$$
C(R, s) d R=D^{(2 d)} \theta(R-s) 2 \pi \zeta d \varsigma
$$

Here $\zeta^{2}=R^{2}-s^{2}$, and the step function is defined according to

$$
\theta(R-s)=1, \text { if } R \geq s, \cdots=0 \text {, if } R<s
$$

The solution of the integral equation (4) for $G(R)$ according to $C(R)$ in eq. (6) reads

$$
\begin{aligned}
& G^{(2 d)}(R)=2 \pi R D^{(2 d)}[1+\theta(R-s)] \\
& \times \exp \left\{-\pi D^{(2 d)}\left[R^{2}+\theta(R-s)\left(R^{2}-s^{2}\right)\right]\right\}
\end{aligned}
$$

In (7) only 2 sheets from Fig. 5 are involved. Consider now Fig. 7 where the test structure to probe the precision of the delta-doped sheets is given in part A of the sketch. Donor-acceptor pairs are formed within and between the sheets.

The corresponding pair distribution function reads now

$$
\begin{aligned}
& G^{(2 d)}(R)=2 \pi R D^{(2 d)}\left[1+2 \sum_{m=1}^{n} \theta(R-m s)\right] \\
& \times \exp \left\{-\pi D^{(2 d)}\left[R^{2}-2 \sum_{m=1}^{n} \theta(R-m s)\left(R^{2}-m^{2} s^{2}\right)\right]\right\}
\end{aligned}
$$

If for any reason not the situation (A) from Fig. 7, but (B) is present, where some doping elements of the central sheet are displaced by one atomic layer (distance $a_{D}$ ), then a series of new pairing chances between donors and acceptors occurs. The new pair distribution function follows with $\theta(R-\beta)=1$ if $R \geq$ $\beta,=0$ if $R<\beta$, from integral equation (4) again. Here $\mathrm{n}$ is the total number of delta-doped superlattice sheets, $j$ is the number of occupied side layers in one direction. Thus, equation (9) is just one special case for $j=1$. Correspondingly could also cases (C) and (D) apply. The case $\mathrm{j}=0$ corresponds to the perfect superlattice without fluctuations. In Fig. 8 function $G^{(2 d)}(R)$ is shown for cases (A) through (D), where each layer is doped with the same amount of donors and acceptors, i.e. $D^{(2 d)}(j)=D^{(2 d)}$. Discontinuities of the otherwise smooth curve appear, whenever the pair separation distance $R$ combines occupied sheets. A first derivative of the function would exactly yield the values $R_{S L}$ and $R_{S L} \pm n a, n=1,2, \ldots$ While this is of less importance here, it will be useful in the later discussion of the DAP-luminescence spectra:

As a final case in the discussion of the pair distribution function the diffusion of doping elements out of the delta-doping sheet is considered. Again Fig. 7 applies where the test structure is resembled in part A. Diffusion of atoms will cause neighboring atomic planes to be occupied by donors and acceptors. The impurity distribution is described by a Gaussian distribution law, i.e. 


$$
\begin{aligned}
& D(z, t)=D^{(2 d)} 2\left(\pi \Phi_{D}^{*} t\right)^{1 / 2} \exp \left[\left(z-i R_{S L}\right)^{2} / 4 \Phi_{D}^{*} t\right](10) \\
& A(z, t)=A^{(2 d)} 2\left(\pi \Phi_{A}^{*} t\right)^{1 / 2} \exp \left[\left(z-i R_{S L}\right)^{2} / 4 \Phi_{A}^{*} t\right]
\end{aligned}
$$

where the variance $\sigma^{2}$ of the distribution due to diffusion corresponds to $\sigma^{2}=2 \Phi^{*} \mathrm{D}, \mathrm{A}, \mathrm{t}$ - time, $\Phi^{*}{ }_{\mathrm{D}, \mathrm{A}}$ - diffusion coefficient of donors of concentration $D$ and acceptors of concentration A, respectively. From here follow in a straight forward manner both $C(R)$ and $\mathrm{G}(\mathrm{R})$.
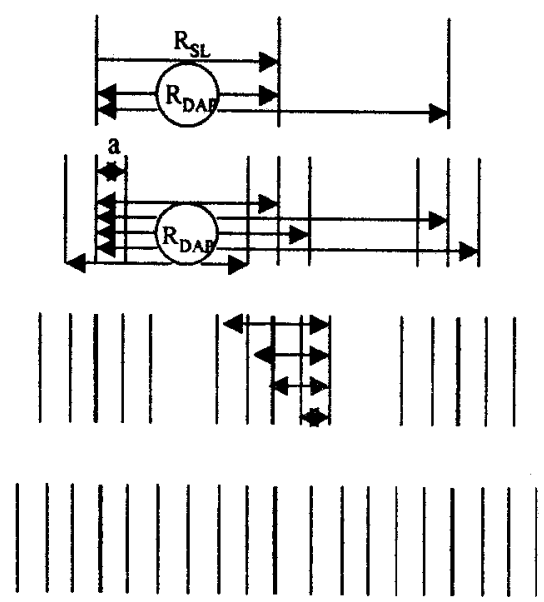

Fig. 7. Superlattice of periodicity RSL with side layers at atomic plane distance $a$.

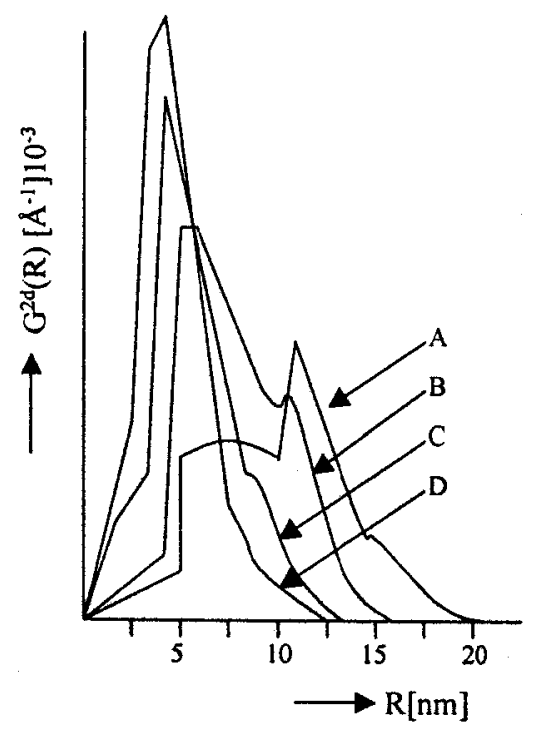

Fig. 8. Pair distribution function for cases described in Fig.7. The superlattice periodicity is $R_{S L}=50 \AA$, the adjacent atomic plane distance of the crystal red is $a=5 \AA$. Curve $D$ represents almost the (3d)-case of the bulk.

\section{Dap-Luminescence in Pair Doped $\Delta \mathrm{i} \Delta \mathrm{i}$ Superlattices}

A donor-acceptor pair once occupied after excitation by an external source, will relax after some time and give raise to a photon of energy.

$h v=E_{\mathrm{g}}-\left(E_{D}+E_{A}\right)+e^{2} / \kappa R$

Here $E_{D}$ and $E_{A}$ are donor and acceptor binding energies, respectively, $E_{g}$ is the gap energy and $\alpha$ is the bulk dielectric constant. The pair separation distance, $\mathrm{R}$, contained in the Coulomb term, relates the measured photon energy to the geometrical arrangement of donors and acceptors in the structure. As $R$ can vary between a nearest neighbor situation up to about twice the Bohr radius of the bound electron or hole, the DAP radiation spectrum is rather broad. Very close pairs produce well resolved discrete recombination lines, which create by incorporation of increasing R-values a more and more smooth but relatively broad main luminescence band. It is this band which is of interest in the context of this paper.

The DAP luminescence intensity $\mathrm{I}(\mathrm{h} v)$ is determined by an expression like

$$
I(h v) \approx R^{2} G(R) \cdot F(R) \cdot P(R)
$$

It depends on the product of the DAP distribution function $G(R)$, the probability of pair occupation $F(R)$, and the probability of recombination $P(R)$. While $G(R)$ in PD- $\Delta \mathrm{i} \Delta \mathrm{I}-\mathrm{SL}$ 's has a quite modified appearance in respect to the bulk case, there is no strong reason for $F(R)$ and $P(R)$ to be changed. They are taken as given for the bulk, depending also in a characteristic way on $\mathrm{R}[17,18]$. In what follows the calculated DAP-luminescence line shapes are given for some cases, using GaAs data for the dielectric constant and Bohr's radius.

(i) Single delta DAP-doped sheet with a probing sheet at a distance $s$ after Fig. 5: The spectrum is shown in Fig. 9. While the photon intensity arising from close pairs is unaffected by the probing layer, a steep jump is seen exactly at values $R=s$ of the sheet under study. The DAP luminescence band for a single sheet (probing sheet at $\mathrm{s} \rightarrow \infty$ ) is also included. The doping concentration of donors is identical in both sheets of $\mathrm{D}^{(2 \mathrm{~d})}=10^{11} \mathrm{~cm}^{-2}$, and $D^{(2 d)} \gg A^{(2 d)}$

(ii) Fluctuations around a delta-DAP-doped sheet with a repetition at RSL of the same structure: Reference is made to Fig. 7, case (B), where instead of just one delta-DAP-doped sheet additionally appear doped sheets at a one-atomic distance $a$ (Fig. 10 ). While the doping concentration of the central sheet is taken to be $\mathrm{D}^{(2 \mathrm{~d})}=10^{11} \mathrm{~cm}^{-2}$, the two side 
atomic layers are varied in doping concentration between $0.1 \mathrm{D}^{(2 \mathrm{~d})}$ (lower curve) and $\mathrm{D}^{(2 \mathrm{~d})}$ (upper curve).

Corresponding to the pairing opportunities between the operating and the probing layer system, discontinuities appear in the luminescence band exactly at the positions $R_{S L}, R_{S L} \pm a$, which by differential amplification would come out as sharp lines. It is interesting to note, that the usual main bulk luminescence band can be accompanied by a sharper second line which might even be stronger in peak intensity than the former one, although it has the same physical nature.

(iii) Diffusion out of a delta-doped atomic-plane: This is a case of particular interest, for the initial diffusion steps of doping elements in semiconductors can be detected. Depending on the diffusion constant and the acting time of thermal excitation $\Phi^{*} t$, neighboring atomic planes are occupied with doping elements, thus reducing the initial sheet doping concentration.

Having Fig. 9 as initial situation $\left(\Phi^{*} t=0\right)$, for $\mathfrak{t} \rightarrow \infty$ the delta-doping structure must convert into the common bulk situation, resembling the main emission band of the donor-acceptor pair luminescence.

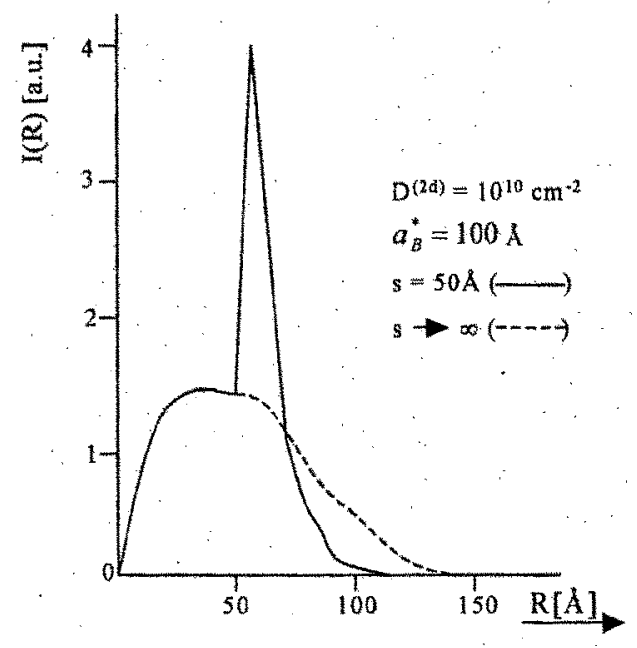

Fig. 9. Single delta-doped plane with a probing plane at a distance $s=50 \AA$.

\section{Summary}

The donor-acceptor-pair luminescence radiation concept is applied to delta-doping layer structures, where the operating delta-doping sheet is accompanied by a probing delta-doping sheet of the same kind. The dependence on $R$ (the donoracceptor-pair separation distance) of the photon energy of DAP transitions provides in such structures for a pronounced change of the DAP luminescence spectrum due to the discrete artificially introduced pairing chances. Once excited to DAP-luminescence, the following and further information is accessible:

(i) Stability and hardness of delta-doping layers.

(ii) Variance of the dopant distribution around the delta-doping sheet as a consequence of i.e. technological insufficiencies during fabrication, segregation, etc.

(iii) Initial steps of impurity diffusion (Diffusion coefficient) [19].

(iv) Spacing of delta-layers.

The appearance of two DAP-main bands in such structures instead of one in bulk DAP-luminescence is an additional feature of this type of SL. Thus, the Pair Doped $\Delta \mathrm{i} \Delta \mathrm{i}$ - Superlattice constitutes a new type of SL's established by the periodic behavior of the DAP distribution.

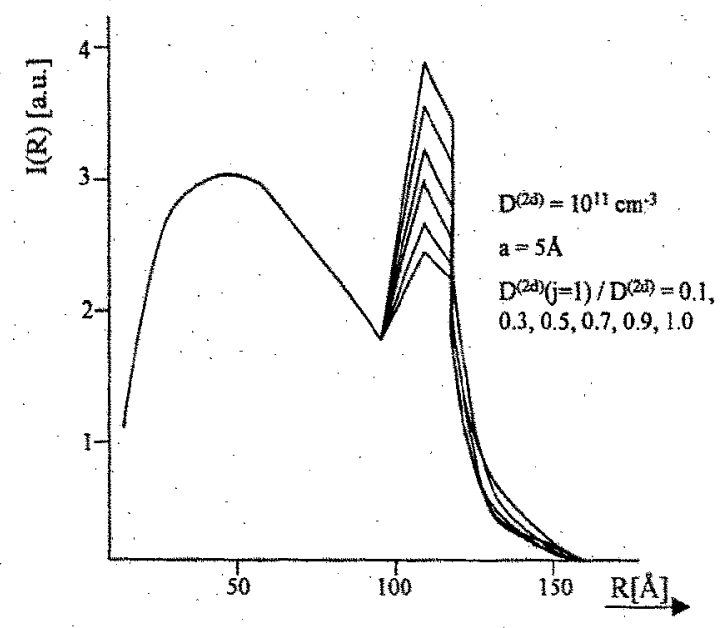

Fig. 10. Effect of fluctuations around a delta-doped plane of $D^{(2 d)}$ with two side layers of distance $a=5 \AA$ and doping concentration between $0.1 \mathrm{D}^{(2 \mathrm{~d})}$ (lower curve) and $\mathrm{D}^{(2 \mathrm{~d})}$ (upper curve).

\section{Referencias}

[1] E. F. Shubert, "Delta-doping of Semiconductors", ISBN 0-521-48288-7, Cambridge Univ. Press, 1996.

[2] C. Weisbuch, R. Dingle, A. Gossard, W. Wiegmann, Solid State Commun. 39, 709 (1981).

[3] S. Adachi, "GaAs and related materials: Bulk semiconducting and superlattice properties", ISBN 981-02-1925-3, World Scientific, 1994.

[4] A. Zehe, in: Crystal Growth and Characterization of Advanced Materials (Christensen et al. editors), World Sci. Publ. Singapore, 1989. 
[5] M. Deveaud, J.Y. Emery, A. Chomette, A., B. Lambert, M. Baudet, Superlattices and Microstructures 1, 205 (1985).

[6] G. Dohler, "Microstructure research", ISBN 3932392-05-9, Ed. Lehrstuhl Microcharact. 1998.

[7] N. Balkan (ed.), "Hot electrons in semiconductors: Physics and devices", ISBN 019-8500-48-0, Osford Univ. Press, 1998.

[8] R. Gaska, M. S. Shur, A. D. Bykhovski, A. O. Orlov, G. L. Snider, Appl. Phys. Lett, 74, 287 (1999).

[9] T. Wang, Y. Ohno, M. Lachb, D. Nakakawa, T. Shirahama, S. Sakaki, H. Ohno, Appl. Phys. Lett. 74, 3531 (1999).

[10] L. K. Li, F Alperin, W. I. Wang, D.C. Look, D.C. Reyworlds, J. Vac. Sci. Technol. B16, 1275 (1998).
[11] C.R. Bolognesi, M.W. Dvorak, D.H. Chow, IEEE Electron Dev. Lett. 19, 83 (1998).

[12] F.F. Harris, R. Murray, C. T. Foxon, Semicond. Sci. Technol. 8, 31 (1993).

[13] S. Sasa, Y. Yamamato, S. Izumiya, M. Yano, Y. Awai, M. Inove, Japn. J. Appl. Phys. 36, 1869 (1997).

[14] K. Ploog, J. Cryst. Growth 81, 304 (1987).

[15] C. Wood, G. Metze, J. Berry, L. Eastman, $J$. Appl. Phys. 51, 383 (1980).

[16] A. Zehe, D. de la Cruz, Superlattices \& Microstructures, 6, 259 (1989).

[17] F. Williams, Phys. Stat. Sol. 25, 493 (1968).

[18] A. Zehe, A. Juarez, G. Röpke, Physica 134B, 374 (1985).

[19] A. Zehe, A. Thomas, "Tecnología Epitaxial de Silicio", ISBN 3-834-1438-2, Ed. ivc Dresden, 2000. 